\title{
Familia y jóvenes en la ecología mediática. Alfabetización digital y el desarrollo de cibercultura en Tijuana
}

Family and young people in the media ecology. Digital literacy and the development of ciberculture in Tijuana

\author{
Gerardo Guillermo León Barrios gleon@uabc.edu.mx \\ http://orcid.org/0000-0002-7332-6146
}

Facultad de Humanidades; Universidad Autónoma de Baja California (México)

\section{Resumen}

Considerando que la nueva Ecología Mediática ha reconfigurado el ambiente sociocultural de las interacciones en donde niños, niñas y jóvenes se desenvolvían tradicionalmente en sus entornos familiares, estos nuevos ecosistemas demandan un acercamiento y propuestas de acción desde la comunicación a través de la alfabetización digital para el desarrollo de 
cibercultura en sus formas de relación familiar; en este caso presentamos un estudio realizado en la ciudad de Tijuana, el cual ha permitido ver posibilidades de este modelo de intervención en este grupo etario y sus formas de socialización.

Palabras clave: Ecología mediática; alfabetización digital; cibercultura; niños; jóvenes; Tijuana.

\begin{abstract}
Considering that the new Media Ecology has reconfigured the sociocultural environment of interactions where children and young people traditionally developed in their family environments, these new ecosystems demand an approach and proposals for action from communication through Digital Literacy for the development of cyberculture in its forms of family relationship; In this case we present a study carried out in the city of Tijuana, which has allowed us to see possibilities of this model of intervention in this age group and its forms of socialization.
\end{abstract}

Keywords: Media ecology; digital literacy; cyberculture; children; youth; Tijuana.

La promesa de la web 2.0 lleva implícita una mirada crítica a casi todas las estructuras de poder. En estas trasformación de escenarios de socialización en internet, parece, hace necesario atender las tensiones generadas por las nuevas formas de organización social que han modificado las funciones, las posiciones y el lugar social de quienes definían tradicionalmente cómo acceder al conocimiento, cómo interactuar con otros, cómo relacionarse con el mundo y crear una cosmovisión. Estos roles han sido claramente trasladados de posición por las posibilidades de relación y comunicación entre usuarios de Internet a través del contacto persona a persona, las redes sociales, las creaciones de colaboración wikis, la posibilidad de compartir lo que se desea por cualquier individuo que cuenta con acceso a la red de redes. Los nuevos patrones de uso y aprendizajes de interacción, incluyendo los sentidos y los significados que se están adquiriendo en sus vida diaria con ello (Buckingham, 2007; Livingstone, 2002; Scolari, 2008) plantean por primera vez en la historia social otras formas de conformación de las estructuras de vida social y formas de vida familiar, esto en tanto niños y 
jóvenes están en las mismas condiciones de interactuar desde un mismo espacio de articulación social como lo es la web 2.0. Por tanto, es incuestionable que la unidad familia como espacio de socialización primaria con niños y/o jóvenes, están experimentando estos reordenamientos en todos los ámbitos de su vida cotidiana (Bochma, 2007), cuyos entornos y procesos de nuevos aprendizajes de socialización desafían los modelos tradicionales de interacción a nivel individual y grupal, con consecuencias en las relaciones sociales más amplias (Cobo y Moravec, 2011) que se han visto afectados por internet (1) y el uso de nuevos dispositivos móviles.

Lo anterior nos lleva a preguntarnos por la labor de la investigación en comunicación, pero sobre todo urge intervenir desde lo comunicacional y educacional en estos escenarios (Buckingham, 2013; Orozco, 2012; Piscitelli, 2010). La nueva generación de "medios digitales interactivos", completamente diferentes en cuanto a sus lógicas de producción y consumo, pero sobre todo de interacción social como las prácticas de comunicación en red e intersubjetivas entre usuarios, le demanda al campo científico un marco de interpretación y acción diferente a la vieja idea de la comunicación de masas.

\section{La ecología mediática y los nuevos entornos de socialización}

Es Niel Postman, y posteriormente otros investigadores quienes han planteado y cultivado el paradigma de la Ecología de los Medios. La Ecología Mediática (o Media Ecology) es más que la mera analogía de la concepción biológica, que se refiere al estudio de los medios como ambientes socioculturales en los cuales viven sus actores sociales, y trata de comprender, fundamentalmente, las relaciones e interacciones que se crean entre todos y cada uno de los elementos del ecosistema al cual pertenecen. En la Ecología Mediática el cambio tecnológico no es "aditivo sino ecológico", esto es, un medio -y desde luego sus contenidos, sus audiencias y prosumidores, así como su carácter institucional y comercial- sostiene una relación de implicación en los universos simbólicos en los que se usan y apropian, de tal manera que todos los elementos -simbólicos- que componen un sistema social son trastocados, afectados y transformados. La llegada de un nuevo medio trae consigo un sin número de cambios, tanto positivos como negativos, por lo que cabe preguntarnos cómo se asumen las destrezas y habilidades para saber utilizar las tecnologías de una manera adecuada, en donde entra en juego factores como las brechas digitales y el analfabetismo digital. Para ello retomamos de los principios medulares de la Ecología de los Medios en donde no importa saber y denunciar 
cómo los medios afectan al actor social, sino que tratamos de comprender la interacción con los medios, y cómo esta facilita o impide nuestras posibilidades de interactividad en esos entornos, estructuras y contenidos. Bajo el paradigma ecológico, como vemos, el actor social tiene un rol diferente, y es entendido como prosumidor, noción de origen inglés que articula las palabras producer y consumer, y que se refiere a que el receptor, la audiencia, o el consumidor asumen simultáneamente los roles de productor-creador y consumidor de contenidos (Benassini, 2014).

De esta manera proponemos entender estos procesos como cambios evolutivos desde el planteamiento de la Media Ecology (Lance, 2015; Islas, 2015; Scolari, 2015) y entendemos a los medios de difusión y a los medios digitales como especies creadoras de ambientes socioculturales y entramados socio-semióticos complejos, generadores de ecosistemas de vida social que modelan la percepción y los procesos cognitivos de los actores sociales. Todo esto generado por dos componentes fundamentales, uno es el cambio en las interacciones individuos-medios digitales-individuos, potenciada en los ambientes de la web 2.0; otro es el avance tecnológico, cuando en un solo dispositivo (como el Smartphone) podemos integrar en una sola experiencia, en el momento deseado y conectado a la red el escuchar noticias, leer el periódico, revisar un correo electrónico, ver contenidos de televisión, tomar fotografías y compartirlas en tiempo real, hablar por teléfono o tener una video-llamada, y que podemos entender como una experiencia social ubicua (Islas, 2009), también Ilamada convergencia cultural (Jenkins, 2006), en la cual el motor de la experiencia mediática del prosumidores el acceso a contenidos según los tipos del estilo de vida que satisfacen sus necesidades de información y entretenimiento, las cuáles puede disfrutar en diferentes plataformas y recursos digitales.

En el marco anterior, consideramos que se requiere de una importante adecuación en la comprensión y acción sobre los medios, sus procesos y características, y al mismo tiempo realizar es necesaria una lectura de los posibles escenarios futuros para contribuir a una mejor relación medios-sociedad en la vida cotidiana, tendiente a la formación de ciudadanos en la construcción de sociedades (Bianchi y Sandoval, 2014).

La experiencia que aquí se presenta forma parte de un proyecto de investigación sobre Medios y Sociedad en la ciudad de Tijuana, Baja California, cuyo objetivo se centra en la exploración de los hábitos de uso y consumo de medios digitales con niños y jóvenes del Sistema Educativo Municipal con el objetivo de contar con una base empírica robusta que permita desarrollar con precisión una alfabetización digital para animar un uso más constructivo y potenciado de los medios digitales en los ambientes sociales familiares. 
Partimos, por lo tanto, del supuesto de que los sujetos sociales aprendemos una serie de conocimientos y habilidades que están directamente en nuestro entorno en tanto nos encontramos en él y en diferentes grados de interacción, y que estos procesos no se dan en un marco de tiempo determinado, sino que son procesos generativos de otros procesos y marcos de conocimiento (Piaget y García, 2009) que se van acompasando y hetero determinando.

Cristóbal Cobo y John W. Moravec plantean la necesidad de un espacio metodológico y analítico que pueda estudiar e intervenir el proceso de adquisición de conocimientos y destrezas fuera de los espacios tradicionales de transmisión de conocimientos tradicionales de los individuos, y lo definen convocando a

\begin{abstract}
construir de manera conjunta un paradigma de educación que resulte inclusivo, que no se anteponga a ningún planteamiento teórico en particular pero que ilumine áreas del conocimiento hasta ahora desatendidas. Aprendizaje invisible no pretende proponer una teoría como tal, sino una metateoría capaz de integrar diferentes ideas y perspectivas. Es por ello por lo que hemos querido denominar un protoparadigma (2011: 22).
\end{abstract}

Con lo anterior, asumimos que los medios digitales constituyen un ambiente, una ecología mediática, que se hacen imperceptibles precisamente por ser parte de este entorno, en su mayoría un entorno informal cotidiano, y que no por estar fuera de los espacios de aprendizajes formales y/o legitimados para "aprender", resultan menos importantes en cuanto al calado de su importancia en los procesos de socialización que se dan día a día, entre sujetos ubicados simultáneamente en diversas y diferenciadas prácticas de interacción.

\title{
Metodología
}

El diseño del estudio y propuesta de intervención de alfabetización digital se ha hecho en dos etapas. La primera consistió en hacer un estudio descriptivo-exploratorio sobre hábitos y prácticas uso de medios digitales relacionado con formas emergentes de interacción en los ámbitos familiares, amical y educativo. En esta primera fase se aplicó un programa metodológico de complementación (Bericat, 1998) con el método cuantitativo y el método cualitativo. En el primero se utilizó la técnica de encuesta con una muestra (no probabilística) de 1776 estudiantes de 3829, con un nivel de confianza del $95 \%$ o superior, con muestras independientes por sistema educativo y grado escolar, obteniendo muestras de más del $40 \%$ 
por nivel educativo, esto es un $46.4 \%$ de la población integrada por estudiantes de tercero a sexto de primaria (diez centros educativos) y secundaria (tres centros educativos). El tipo de preguntas para estas gráficas se basó en una escala tipo Likert.

\begin{tabular}{|l|l|l|l|}
\hline Nivel educativo & Población & Muestra & $\%$ \\
\hline Primarias & 1673 & 477 & 28.51 \\
\hline Secundarias & 1701 & 786 & 46.21 \\
\hline
\end{tabular}

Tabla 1. Diseño de muestra. Estudio de hábitos de uso y consumo medios digitales,

Secretaría de Educación Pública Municipal, Tijuana.

Con el segundo método se utilizó la técnica de entrevistas grupales a niños, niñas y jóvenes con una muestra a pertinencia segmentado por centro escolar (primaria y secundaria) y por grado de cada nivel (tercero, cuarto, quinto y sexo para el caso de primaria; y primero, segundo y tercero para el caso de secundaria), con una guía de entrevistas estructurada bajo tres ámbitos (familiar, amical y educativo) fundamentales en la formación social de los niños, las niñas y los jóvenes con preguntas descriptivas y proyectivas para conocer la prácticas sociales en donde se encuentran medios digitales como parte de su cultura mediática y equipamiento.

\section{Resultados del estudio}

Hallazgos en cifras 


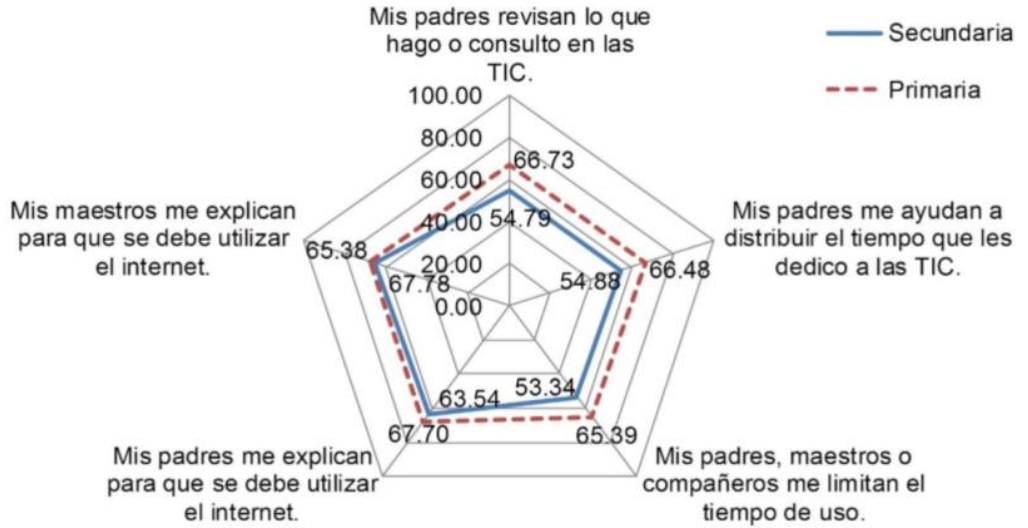

Gráfico 1. Acciones de participación de padres y maestros con niños y jóvenes en uso de medios digitales.

En las siguientes tres gráficas, la estadística nos hace visible que en los tres ámbitos de interacción familia, escuela y espacio individual, los niños, niñas y jóvenes cuentan con habilidades y destrezas de acción según diferentes objetivos de su vida diaria. Esto nos permite visibilizar que hay una serie de aprendizajes que no necesariamente pasaron por los espacios legitimados para tal fin. En el caso del Gráfico 1, nos representa que la participación de padres de familia y maestros está casi en el porcentaje y pasa, por poco, el $50 \%$ en la toma de decisiones; esto es, hay menos centralidad en estos acompañamientos por parte de las figuras tradicionales de autoridad.

En el siguiente gráfico, se representan los usos habituales de medios digitales, así como de acciones y tomas de decisión en donde en un porcentaje alto (mayor al 50\%) en las capacidades de autonomía para interactuar y usar medios digitales, sobresalen el trabajo y la resolución de retos cotidianos con los pares. De manera específica, las acciones asociadas a actividades escolares están soportadas por aprendizajes entre pares y de manera colaborativa. 


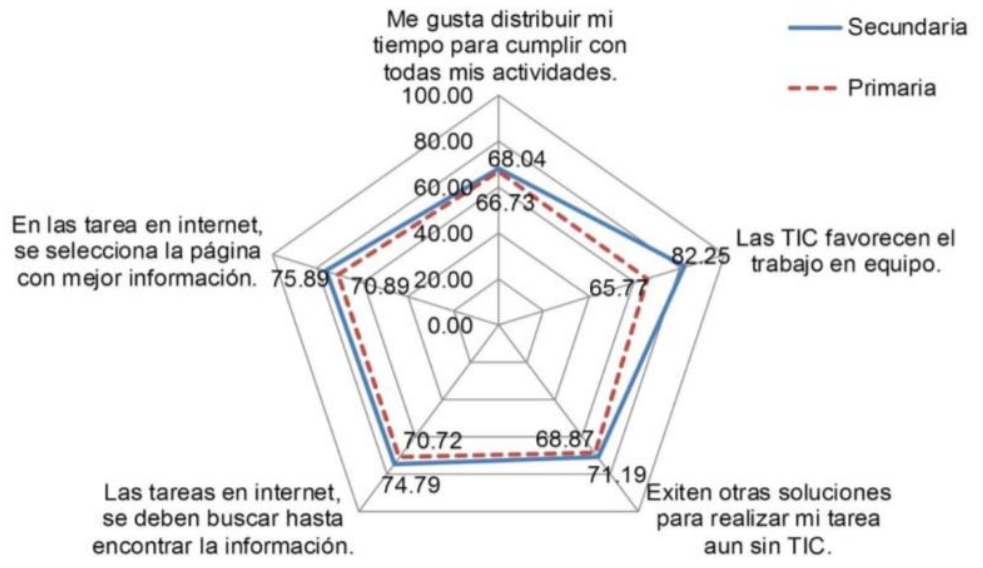

Gráfico 2. Uso habitual de medios digitales decididos por los mismos niños, niñas y jóvenes.

En la última tabla, es de especial importancia el grado de conocimiento de las implicaciones sociales en cuanto a su participación en medios digitales, la vida social tiene tintes más constructivos en tanto el niño, la niña o el joven puede interactuar con otros y él mismo en el ciberespacio, lo cual hace visible que formas de interacción de tipo participación y ciudadanización parece están emergiendo por el sentido de colaboración en otro espacio público en donde es importante y visible el mismo individuo.

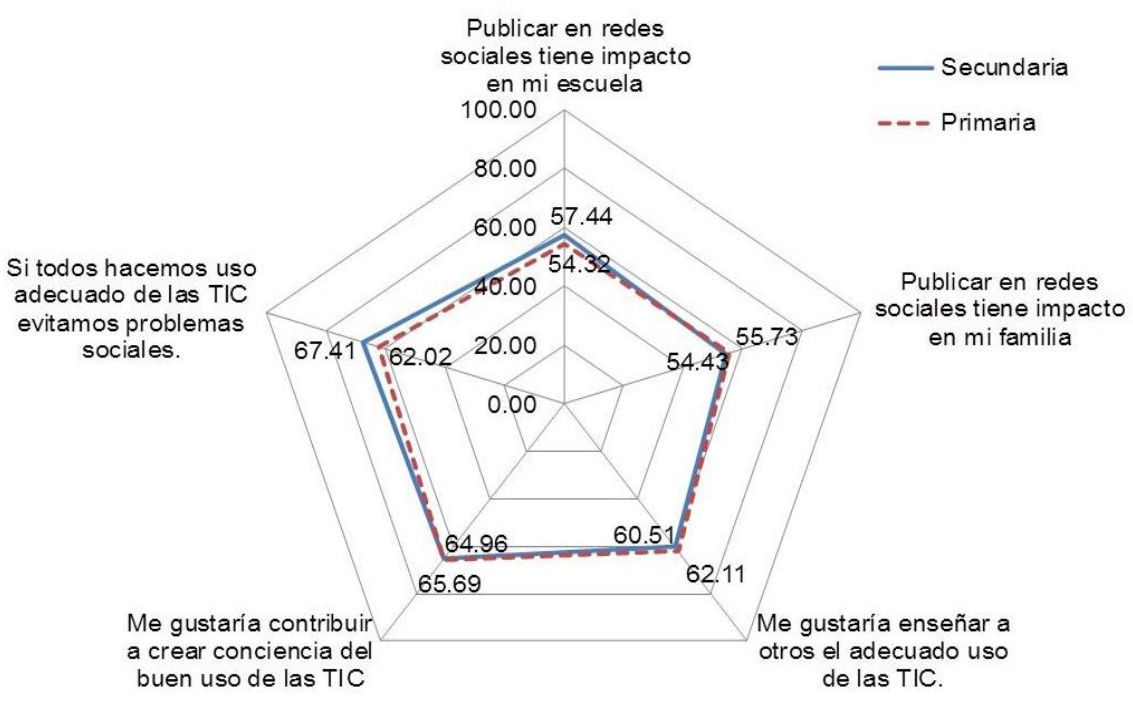

Gráfico 3. Implicaciones sociales de uso de medios digitales en niños y jóvenes. 


\section{Hallazgos cualitativos}

Como síntesis analítica de los resultados de las entrevistas grupales, presentamos siete argumentos sobre los cuáles podemos comprender los entornos en los que niños, niñas y jóvenes se ven inmersos.

a. Los entornos de interacción social se han expandido con los medios digitales para niños, niñas y jóvenes del SEPM, los espacios tradicionales de socialización son superados en formas y número y se ven a los medios digitales como nuevas oportunidades para la vida social, con un efecto importante en la sociedad en general.

b. Los hábitos de uso de los medios digitales son un espacio imprescindible y altamente complementario para la formación del sentido de pertenencia, el desarrollo de su identidad, la formación de valores, en los cuales la familia y la escuela están completamente desplazados, quizá a un segundo orden, en cuanto al lugar que ocupan en la mediación social y procesos de socialización, pero sin desaparecer por completo.

c. Los contextos familiares, sociales y educativos son un factor muy importante en el desarrollo de hábitos de uso de medios digitales. El contexto familiar de los niños marca una gran diferencia en el tipo de acceso a los medios, así como el sentido de su uso. No se usan los medios digitales en situaciones y contextos donde estos no son centrales e importantes como mediaciones tecnológicas para el entretenimiento, la información y la socialización.

d. Los medios tradicionales, específicamente la TV, sigue siendo un medio importante que se ve en los hogares, los contenidos de telenovelas y noticieros aparecen como eje de las rutinas de la vida cotidiana, y los hijos llegan a ver y seguir los contenidos acompañando a los padres, y se combina cada vez más el uso de medios digitales.

e. La vida social familiar se ve transformada por el uso de medios y nuevos medios por parte de los padres. La interacción con los hijos es escasa, no hay momentos y espacios de construcción de vínculos sólidos, o han cambiado de sentido y significado para la construcción de vida social.

f. Los padres de familia experimentan una sensación de confusión entre dos aspectos: con idea de lo público y los privado de la vida social familiar y la de los hijos en medios digitales; y dos, entre la importancia de su uso bajo el supuesto de que representan un acceso a lo moderno y aseguran mayor nivel educativo pero también representan un riesgo en cuanto al acceso a contenidos sin control y el contacto con personas desconocidas. La distinción tradicional de 
estos aspectos se ha dislocado, generando gran incertidumbre sobre la educación familiar con los hijos, pero no se sabe cómo entenderla y practicarla.

g. Hay un cambio muy visible en cuanto a las jerarquías sociales que anteriormente pautaban el acceso, uso y sentido de los medios en general, y con los medios digitales; este proceso están estructurado por los niveles de participación en los ámbitos familiar, escolar y amical.

\section{Participación y consenso en las interacciones sociales y medios digitales}

Es claro que los niños, niñas y jóvenes han desarrollado habilidades para la solución de una serie de situaciones de su vida diaria de manera muy eficaz. En eje de estos aprendizajes está la participación y la colaboración entre pares. Hay una notable diversidad de experiencias compartidas dentro de subgrupos específicos, determinados por factores sociales, psicológicos y culturales. La enseñanza de la informática en las escuelas es muy elemental y limitada. Es importante reconfigurar la organización social institucional que genera divisiones sociales existentes, estructuras sociales muy empobrecidas socioculturalmente, si tomamos en cuenta que el mundo que experimentan los niños, las niñas y los jóvenes en los medios digitales les gratifica profundamente, y está más cerca, sin duda, de sus necesidades de socialización y usos de información. Parece importante reapropiar y resignificar el sentido de los usos de medios digitales. El ámbito familiar parece sumamente importante si se articula con el ámbito educativo y se articula a procesos de aprendizaje horizontales con niños y jóvenes-familiaescuela, los niños, las niñas y los jóvenes también poseedores de conocimientos y habilidades de los que los adultos pueden reaprender de manera significativa.

\section{La alfabetización digital trascendida: gestión del consenso familiar}

\section{De la educomunicación a la alfabetización digital}

La Alfabetización Mediática y la Alfabetización Digital, Ilamada inicialmente Educación para los Medios (EPM) tiene sus inicios en países como España, Francia, Inglaterra y Canadá, entre otros, que junto con la UNESCO (1984), implementan una línea de intervención a manera de respuesta ante la preocupación por las posibles repercusiones socioculturales, debido al poder simbólico mostrado por los medios masivos de difusión y sus mensajes audiovisuales. Estos primeros esfuerzos intentaron convertirse en una forma de contrarrestar la influencia sobre las grandes audiencias y la formación crítica de niños y jóvenes a través de la "educomunicación", 
perspectiva que representaba una educación del público para un consumo juicioso, informado y educado en cuanto a lo que representaba la cultura de masas y los contenidos de los medios de comunicación (Livingstone y Bober, 2005). En las discusiones y debates sobre este modelo de intervención desde la comunicación y educación, se parte del principio de que no se ha logrado que población en su conjunto tenga los niveles mínimos de alfabetismo tradicional, sobre todo en la lecto-escritura, lo que implica, desde estos puntos de vista, problemas en cuanto a los "accesos" al no contar con una herramienta básica de desarrollo moderno occidental. En otras reflexiones afines a la premisa anterior, pero contradictoriamente, se ven a las tecnologías como un excelente recurso y solución para la adquisición de conocimiento, sólo que deben ser "bien" aplicados; todo esto sin considerar que las TIC y los medios digitales no son por sí mismos la solución al retraso de alfabetización básica, sino de una posible metodología de incorporación y asimilación en todos los ambientes de la vida cotidiana de niños, jóvenes y adultos. Estas posturas deben de ser matizadas, sobre todo en el ambiente actual de las TIC, los nuevos medios, los medios digitales, así como todos sus recursos y lenguajes multimediales y transmediales, debido a que los criterios para pensar en la representación y los accesos a información no son conducidos y/o acompañados por métodos y recursos que contrarresten afectaciones en la interacción, confundan en el mar de información o generen sensaciones de bienestar y placer por el simple hecho de contar con tecnología de alta gama y con acceso a internet (Livingstone, 2003).

Sonia Livingstone (2008) distingue un importante debate en el tema de la alfabetización, y que tiene que ver con los alcances de este modelo de intervención sobre los sujetos, un tema que trastoca criterios de ética, y tiene que ver con que si la alfabetización mediática se debe considerar un beneficio en el orden individual, que consigue cada sujeto con la incorporación de los elementos "aprendidos" para el manejo y conocimiento de los medios; o por el contrario, la alfabetización mediática representa una acción sociocultural que socializa el "aprendizaje" con respecto a la mirada y reflexión crítica hacia medios de comunicación y sus contenidos, y con ello lograr un mejor resultado. Las dos posturas parecen sugerentes, pero creemos que ambos procesos son claramente complementarios.

A partir de esto, Livingstone (2004) destaca dos importantes ejes de acción y apuesta en cuanto a la alfabetización. La llamada alfabetización informativa, considerada también en la declaración de Praga (2003) una condición para acceder activamente a la "Sociedad de la Información", y que atiende procesos de aprendizaje sobre "uso y necesidades informativas", mediante el desarrollo de destrezas para "identificar, localizar, evaluar, organizar y crear" formando sujetos que tengan las competencias para "hacer uso y comunicar información para 
abordar temas y problemas", una habilidad que se puede cultivar para intervenir en el mundo (Linvignstone 2004). El otro terreno de acción, que ha detonado amplios debates, propuestas y experiencias de acción es la alfabetización mediática, que se entiende de manera fundamental como los aprendizajes desarrollados para "comprender" a los medios de difusión como "ventanas representan al mundo", y/o en su caso a través de los cuáles se puede "representar el mundo" desde la visión del actor lego.

En ambos casos, aún con las divergencias conceptuales y metodológicas, se tienen apuestas de intervención que desembocan en una dirección, que es dotar a las personas de habilidades para "usar" en su vida cotidiana a las TIC, los viejos y los nuevos medios, las redes sociales y otras plataformas, como recursos para una relación medios-sociedad que promuevan una calidad de vida tanto en lo individual como en lo colectivo.

Fundamentado en estos principios, se asume que el desafío de la alfabetización digital, como un programa científico-académico, es plantear, articular y trascenderla alfabetización informativa y la alfabetización mediática, lo cual considera que esta acción de intervención empodera a los sujetos sociales y construye ciudadanía en el nuevo orden social digital con el desarrollo, asumiendo derechos y responsabilidades en acciones individuales y colectivas (Buckingham, 2000), como ilustramos en la Tabla 2.

\begin{tabular}{|c|c|c|c|c|}
\hline $\begin{array}{l}\text { Nivel de } \\
\text { alfabetiza- } \\
\text { ción }\end{array}$ & $\begin{array}{c}\text { Tipo de } \\
\text { alfabetización }\end{array}$ & $\begin{array}{c}\text { Aprendizaje- } \\
\text { adquisisción de } \\
\text { conocimiento en } \\
\text { las TIC y con las } \\
\text { TIC }\end{array}$ & $\begin{array}{l}\text { Desarrollo de } \\
\text { pensamiento } \\
\text { crítico-creativo }\end{array}$ & $\begin{array}{l}\text { Acción social } \\
\text { constructiva }\end{array}$ \\
\hline I & $\begin{array}{l}\text { Alfabetización } \\
\text { tecnológica }\end{array}$ & $\begin{array}{l}\text {-Se logra la } \\
\text { oportinidad de } \\
\text { acceso para la } \\
\text { reducciòn de la } \\
\text { "brecha digital". } \\
\text {-Se desarrollan } \\
\text { habilidades básicas } \\
\text { de uso de TIC } \\
\text {-Se adquieren } \\
\text { destrezas báscias }\end{array}$ & $\begin{array}{l}\text { Desarrollo de } \\
\text { competencias } \\
\text { básicas } \\
\text { Destrezas de uso } \\
\text { de herramientas y } \\
\text { textos } \\
\text { Destrezas de } \\
\text { entrada y salida } \\
\text { Desarrollo de } \\
\text { habilidades multi- }\end{array}$ & $\begin{array}{l}\text { Se empodera en } \\
\text { el acceso a la } \\
\text { distribución de } \\
\text { servicio y uso } \\
\text { de servicio, } \\
\text { infraestructura, } \\
\text { herramientas de } \\
\text { internet }\end{array}$ \\
\hline
\end{tabular}




\begin{tabular}{|c|c|c|c|c|}
\hline & & $\begin{array}{l}\text { de navegación en } \\
\text { la web }\end{array}$ & tareas & \\
\hline II & $\begin{array}{l}\text { Alfabetización } \\
\text { mediática }\end{array}$ & $\begin{array}{l}\text {-Se conocen los } \\
\text { riesgos del peligro } \\
\text { que implica internet } \\
\text {-Se aprenden } \\
\text { criterios de } \\
\text { seguridad } \\
\text {-Se logran } \\
\text { habilidades de } \\
\text { reflexión y síntesis } \\
\text {-Se desarrollan } \\
\text { destrezas de } \\
\text { resolución de } \\
\text { problemas }\end{array}$ & $\begin{array}{l}\text {-Se toman } \\
\text { decisiones } \\
\text {-Se crean acciones } \\
\text { en red } \\
\text {-Se aplican } \\
\text { habilidades } \\
\text { creativas } \\
\text {-Se desarrollan } \\
\text { habilidades de } \\
\text { apropiación de } \\
\text { información y } \\
\text { acciones de red } \\
\text {-Se forman } \\
\text { capacidades de } \\
\text { juicio y } \\
\text { dicernimiento sobre } \\
\text { información y } \\
\text { acciones en } \\
\text { internet }\end{array}$ & $\begin{array}{l}\text {-Se empodera } \\
\text { en la } \\
\text { comprensión y } \\
\text { la creación }\end{array}$ \\
\hline III & $\begin{array}{l}\text { Alfabetización } \\
\text { digital }\end{array}$ & $\begin{array}{l}\text {-Hay desarrollo de } \\
\text { haibilidades de } \\
\text { conocimiento } \\
\text { compartido } \\
\text {-Se desarrollan las } \\
\text { destrezas de una } \\
\text { conciencia social e } \\
\text { identidad, } \\
\text { individual y } \\
\text { colectiva }\end{array}$ & $\begin{array}{l}\text {-Se desarrollan } \\
\text { acciones de } \\
\text { cognición } \\
\text { disitribuida } \\
\text {-De aplican } \\
\text { acciones usos y } \\
\text { generación de } \\
\text { información y de } \\
\text { investigación- } \\
\text { creación }\end{array}$ & $\begin{array}{l}\text {-Se empodera } \\
\text { en } \\
\text { conocimientos y } \\
\text { sus aplicaciones } \\
\text { sobre los } \\
\text { medios digitales }\end{array}$ \\
\hline
\end{tabular}

Tabla 2. Niveles y operaciones de la alfabetización tecnológica, mediática y digital. Fuente: elaboración propia con información de Buckingham (2000). 
De la alfabetización digital al desarrollo de cibercultura en la socialización familia, niños, niñas y jóvenes

Los nuevos entornos digitales son en el centro de la vida social de niños, niñas y jóvenes, y sitúan en un lugar diferente a las instituciones que tradicionalmente acompañaban y/o mediaban los aprendizajes. De acuerdo con Pérez Tornero, la alfabetización digital demandada por estas circunstancias apela a una participación activa y creativa de la familia y la escuela, principalmente, bajo la idea de articular un proceso de acompañamiento y participación en la formación de nuevos ciudadanos; con esto nos referimos a que necesario repensar las maneras en que la autoridad paternal de la formación de hijos y la autoridad educativa para formar individuos debe contribuir, a la par de ellos (niños, niñas y jóvenes), es decir, aprender de sus formas y aprendizajes que han desarrollado en su espacios de socialización digital para formar no únicamente individuos que usen y produzcan críticamente contenidos, sino que constituyan plataformas sociales de aprendizajes mutuos (adultos y niños, niñas y jóvenes) para tener ciudadanos con capacidad de vinculación y articulación en el tejido social.

La propuesta de diseño y aplicación de los talleres y actividades de alfabetización digital que proponemos se encuentra fundamentado en el programa metodológico de la Ingeniería Comunicación Social desarrollada por Jesús Galindo Cáceres (2006, 2011a, 2011b, 2014, 2015), específicamente en la metodología de intervención desde la comunicación y el desarrollo de cibercultura sobre los sistemas y las configuraciones de relación a partir de formas meméticas construidas en sistemas de información, o en su caso, en sistemas de comunicación. Con ello se puede ver la posibilidad de conocer en dónde se puede intervenir en cuanto a aspectos sistémicos de interacción, de procesos, de dinámicas y de prácticas de relación entre individuos en un sistema o varios sistemas, bajo cinco niveles de desarrollo cibercultural:

a. Nivel de contacto. Nivel mínimo de relación entre dos o más individuos de un sistema o sistemas. No hay ningún tipo de intención y compromiso por las partes que busque modificar a alguno o algunos de los elementos o de los sistemas en juego.

b. Nivel de interacción. Este es un nivel de relación medio. Las acciones tienen una intencionalidad que buscan afectar a los elementos y a los sistemas en juego. Las relaciones implican diferentes grados de intención, compromiso y afectación. Podemos hablar aquí ya de un primer grado de comunicación y articulación. 
c. Nivel de conexión. Grado de relación alto. Hay acuerdo entre los individuos. Se comparte la intencionalidad, el compromiso y se acepta alguna forma de afectación dentro de un espacio común y de una relación. El nivel de comunicación es medio. Hay interés en sostener la interacción y pasar a un estado diferente, pero no más.

d. Nivel de vínculo. Grado de relación alto entre individuos. Supone un nivel de acuerdos altos en los que se define, por las partes, la intencionalidad, el compromiso y las formas de afectación, por lo tanto hay un acuerdo por la sustentabilidad y continuidad de la relación en tiempo y espacio.

e. Enacción. El entorno, los sistemas y los individuos entran en una relación compleja de colaboración en la configuración de los compromisos y del entorno; todos colaboran con un compromiso no necesariamente tácito, tampoco explícito, y suceden transformaciones y cambios para bien del sistema.

Con el desarrollo de cibercultura como modelo de intervención en el diseño de alfabetización digital con niños, niñas y jóvenes en el Sistema Educativo Municipal de Tijuana, planteamos que la alfabetización digital debe de superar el nivel 1 y 2 de la Tabla 2, y apostando por una acción social constructiva de empoderamiento sobre las capacidades de los medios digitales en el marco de las posibilidades que permite la web 2.0 y su ecología mediática, lo que significa que los aprendizajes y adquisición de conocimientos de uso de medios digitales para compartir conocimiento y desarrollo de destrezas para una conciencia social, la identificación de una identidad individual y colectiva, así como el desarrollo de pensamiento crítico-creativo para acciones de cognición dsitribuida, socialmente compartida y de creación social, son sólo elementos y aprendizajes muchas veces desarollados y aprendidos por los mismos niños, niñas y jóvenes.

\section{Conclusiones y discusión: puntos de discusión de la alfabetización digital desde la cibercultura}

Un elemento que resulta clave es que los jóvenes participantes saben diferenciar los medios de comunicación existentes. Sin embargo, les resulta más práctico y accesible usar las plataformas de redes sociales para informarse de los hechos ocurridos. 
Es claro que los jóvenes utilizan los medios de comunicación como herramientas de entretenimiento, y nos los consideran herramientas de aprendizaje, sin embargo es innegable que aprenden todo un universo de sentidos y significados. Muchos no lo hacen de manera consiente porque nacieron sumergido en este mundo multi-tasking, como la capacidad de hacer varias tareas con diversas plataformas y lenguajes, de manera simultánea.

Las acciones de colaboración es un tema a resultar. Los jóvenes realizan diversas acciones de colaboración con sus pares, y resuelven una gran cantidad de situaciones de su vida diaria, sin embargo no se dan cuenta del potencial que tienen estos aprendizajes para expandirlos a muchos otros ámbitos de la vida social; por otro lado estas prácticas deberían tener una mayor inclusión en el ámbito social escolar, ya que en un ejercicio se observó la gran capacidad constructiva, como por ejemplo en una práctica cada uno de los participantes propuso cómo ayudarían a la sociedad mediante las redes sociales.

- Hace falta que alguna entidad institucional o un comité ciudadano y/o académico participe en la regulación de la actividad de jóvenes en cuanto a consumo de contenidos y uso de nuevos medios. Los padres de familia no tienen recursos y criterios suficientes para hacer frente a este desafío en casa, o muchas veces ellos son usuarios sin comprender las implicaciones y la debida importancia por su impacto sociocultural. No se cuenta con elementos para formar un criterio más crítico en lo que consumen, dada la susceptibilidad ante los riesgos de todo tipo en Internet. Resulta necesario, en este sentido, que la interacción y participación con los padres sea eficaz y continua, dado que ellos son parte, o empiezan a ser parte de este escenario (Livingstone, 2003).

- En cuanto al ambiente escolar como espacio social, se observa una urgente necesidad de incluir estos temas en el aula de manera actualizada, no como un contenido, sino como un recurso de formación de individuos, si bien los alumnos ya están "familiarizados" con la tecnología, pero el entorno escolar podría reforzar la alfabetización digital, no cumpliendo con un requisito de "enseñanza" contenidos.

- Se hace ineludible utilizar de forma constructiva los medios de difusión, incluyendo a los nuevos medios, como una de las prioridades y necesidades actuales en materia de educación tanto a niños y niñas como jóvenes. Buckingham (2008) señala la implicación del uso de medios digitales (videojuegos, computadora, internet y ahora redes sociales) en una serie de "procesos informales de aprendizaje", en donde es posible una relación más "democrática" y constructiva profesores, estudiantes y, agregaríamos a los padres de familia.

- La utilización de los dispositivos móviles debe ser por medio de una guía y/o con conocimientos previos que ayuden a los niños y jóvenes a discernir de materiales y contenidos 
que no son constructivos o que reproducen acciones o sucesos negativos para sus grupos. De tal manera que en la Ecología Mediática actual los prosumidores están creando y consensando prácticas de comunicación y aprendizaje que no tiene nada que ver con los modelos tradicionales de enseñanza-aprendizaje, que implican un sin fin de elementos de generación de procesos de conocimiento (Orozco y Franco, 2014). Por lo tanto, se tiene que equipar cognitivamente a los jóvenes para que comprendan y observen de manera más crítica los medios digitales, ya que se pueden aplicar únicamente de un modo "funcional o instrumental" (Buckingham, 2005), y sin recuperar esto otros aprendizajes colectivos.

- El joven participa en su Ecología Mediática fundamentalmente para fines de entretenimiento, incluyendo la interacción con sus pares, por lo que se debe reconsiderar elementos en toda su capacidad configuradora y reconfiguradora en la formación de individuos en casi todas las dimensiones de su vida diaria.

\section{Notas}

(1) Según el último estudio de World Internet Proyect (WIP), en su reporte de 2013 sobre hábitos y percepciones de los mexicanos sobre internet y diversas tecnologías asociadas (2014), a nivel internacional, México se encuentra en el lugar número 11 entre los países con mayor número de usuarios de internet en el mundo, con 59.2 millones. Del dato anterior, según el mismo reporte, poco más de 9 millones de usuarios son nativos digitales, esto es, son niños menores de 12 años que nacieron y han desarrollado toda su vida individual y social en ambientes digitales, con Tecnologías de Información y Comunicación (TIC) relacionadas con internet. De ese total de usuarios (59.2 millones) de internet en nuestro país, $16 \%$ tiene entre 3 y 11 años de edad y $42 \%$ tienen entre 12 y 25 años.

\section{Bibliografía}

Benassini, C. (2014). De audiencias a prosumidores. Acercamiento conceptual. Revista Luciérnaga, 6(12), pp. 16-29. Facultad de Ciencias de la Comunicación, Medellín.

Bericat, E. (1998). La integración de los métodos cuantitativo y cualitativo en la investigación social. Barcelona: Ariel.

Bianchi, M. P. y Sandoval, L. R. (2014). Habitar la red. Comunicación, cultura y educación en entornos tecnológicos enriquecidos. Argentina: Editorial Universitaria de la PatagoniaEDUPA.

Bochma, J. (2007). Generación Einstein. Más listos, más rápidos, más sociable. Barcelona: Ediciones Gestión 2000. 
Buckingham, D. (2000). The Making of Citizens: Young People, News and Politics. Londres: Routledge.

Buckingham, D. (2003). Media Education: Literacy, Learning and Contemporary Culture. UKUSA: Polity Press.

Buckingham, D. (2005). Educación en medios. Alfabetización, aprendizaje y cultura contemporánea. España: Paidós Comunicación.

Buckingham, D. (2007). Beyond Technology: Children is Learning in the Age of Digital Culture. Cambridge UK: Polity Press.

Buckingham, D. (2008). Más allá de la tecnología. Aprendizaje infantil en la era de la cultura digital. Manantial, Buenos Aires.

Cobo Romaní, C. y Moravec, J. W. (2011). Aprendizaje Invisible. Hacia una nueva ecología de la educación. Barcelona: Laboratori de Mitjans Interactius/Publicacions i Edicions de la Universitat de Barcelona.

Galindo Cáceres, L. J. (2006). Cibercultura. Un mundo emergente y una nueva mirada. Toluca: CNCA- Instituto mexiquense de la cultura.

Galindo Cáceres, L. J. (2011a). Ingeniería en Comunicación Social y Deporte. México: INDECUS.

Galindo Cáceres, L. J. (2011b). Ingeniería en Comunicación Social y Promoción Cultural. Sobre Cultura, Cibercultura y Redes Sociales. Argentina: Homo Sapiens, Universidad Nacional del Rosario, Benemérita Universidad Autónoma de Puebla.

Galindo Cáceres, L. J. (2014). Ingeniería en Comunicación Social. Hacia un Programa General. Puebla: Instituto de Ciencias de Gobierno y Desarrollo Estratégico-Benemérita Universidad Autónoma de Puebla.

Galindo Cáceres, L. J. (coordinador). (2015). Ingeniería en Comunicación Social y Familia. Cuadernos Artesanos de Comunicación, 79, Latina, La Laguna, Tenerife.

Islas, O. (2015). La ecología de los medios: metadisciplina compleja y sistémica. Palabra Clave, 18(4), pp. 1057-1083. doi: 10.5294/pacla.2015.18.4.5.

Islas, O. (2009). La convergencia cultural a través de la ecología de medios. Comunicar, XVII(33), pp. 25-33, Grupo Comunicar Huelva, España.

Jenkins, H. (2006). Convergence Culture: Where Old and New Media Collide. New York: New York University Press.

Livingstone, S. (2002). Young People and New Media: Childhood and the Changing Media Environment. Londres: Sage. 
Livingstone, S. (2003). The changing nature and uses of media literacy. Media@LSE Electronic Working Papers, 4. Recuperado de http://eprints.Ise.ac.uk/13476/

Livingstone, S. (2004). What is media literacy? Intermedia, 3(32), pp. 18-20, International Institute of Communications, UK.

Livingstone, S. y Bober, M. (2005). UK Children Go Online. Economic and Social Research Council, London. Recuperado en: http://eprints.Ise.ac.uk/395/1/UKCGOsurveyreport.pdf Livingstone, S.; van Couvering, E. y Thumim, N. (2008). Converging traditions of research on media and information literacies: Disciplinary, critical and methodological issues. En Leu, D.J.; Coiro, J.; Knobel, M. y Lankshear, C. (eds.). Handbook of Research on New Literacies (pp. 103-132). Nueva Jersey, Estados Unidos: Lawrence Erlbaum Associates.

Organización de las Naciones Unidas para la Educación, la Ciencia y la Cultura (UNESCO) (1984). Educación para los medios. Recuperado de https://bit.ly/2esS5c8

Orozco Gómez, G. y Franco, D. (2014). Al filo de las pantallas. Guía didáctica para padres y maestros. Buenos Aires: La Crujía.

Scolari, C. (2015). Ecología de los medios Entornos, evoluciones e interpretaciones. España: Gedisa.

Pérez Tornero, J. M. (2008). La sociedad multipantallas: retos para la alfabetización mediática. Comunicar, XVI(31), pp. 15-25, Grupo Comunicar, España.

Piaget, J. y García, R. (2009). Hacia una lógica de las significaciones. España: Gedisa.

Scolari, C. (2008). Hipermediaciones. Elementos para una teoría de la comunicación digital interactiva. España: Gedisa.

Strate, L. (2015). Estudiar los medios como medios: McLuhan y el enfoque de la ecología en los medios. En Scolari, C. Ecología de los medios Entornos, evoluciones e interpretaciones (pp. 147-164). España: Gedisa.

The Prague Declaration (2003). "Towards an Information Literate Society". Recuperado de http://www.unesco.org/new/fileadmin/MULTIMEDIA/HQ/CI/Cl/pdf/PragueDeclaration.pdf

World Internet Proyect (2014). Reporte Especial (5ta. edición). Estudio 2013 de hábitos y percepciones de los mexicanos sobre Internet y diversas tecnologías asociadas. Centro de Desarrollo de la Industria de Tecnologías de Información en México y Tecnológico de Monterrey CEM, Proyecto Internet. 